\title{
Digit-simulate of Reflect Highlights Model from Submarine
}

\author{
Xu Xiaohu \\ Science and Technology on Underwater Acoustic Antagonizing Laboratory, Beijing, China \\ Xxh.seri@163.com
}

Keywords: reflect highlights model, digital simulation

\begin{abstract}
This paper presents that the reflection signal from a submarine can be seen as the sum of a few strong highlights and a large of weak random highlights background. Using interpolation method can create every hydrophone's signal in a plot array from a single hydrophone's output. At last the time domain signal of the center of an array is simulated.
\end{abstract}

\section{Introduction}

As the developments of under water anti-torpedo technology, there is more challenge to the modern torpedo self-homing system. the size distinguish of a real target and all jammers is obviously. All jammers are in small size. The highlights information is the basis of volume target estimation. There are many simulation study in the target length estimation by use of echo light distribution characteristic(article[1][2]). But the digit simulation of echo highlights is singular. The paper build up the submarine echo model benefit to torpedo work condition, then give the calculation method of time signals on torpedo's array spots, and give the simulation result at last.

\section{Target highlight model}

The torpedo self-homing system normally work on the higher frequency(20-40kHz). In this condition, any complex target's echo is compose of many sub-echo added. Every sub-echo can treat as the reflect wave of some scatter point. This scatter is highlight point, which is maybe real or equivalent highlight. The any complex target's echo is the compose of many scatter highlight, every scatter point create a highlight echo, the total echo wave is the interference of all the highlight.

There are three typical conditions that the object under water create highlight:

Mirror Reflect Highlight. Because of the difference of impedance between the submarine material and the ocean water, The sound wave will be strongly reflected at some special angle of incidence when echo from the smooth surface having a large size than the wavelength. This kind of highlight is the most strongly in most scale angle of incidence target relative bearing. The different type target has a different construction of highlight echo because of the difference of appearance shape and geometry, which is target of sound fuse. The interval of highlight is relative to the value of the incidence because of the highlight has some relationship with the incidence angle.

Angular Scattering[5]. There are some linear edge on the submarine, such as bulbous bow and screw propeller. When the ridge vertical with the beam, and the length of the ridge is greater than the irradiated sound wavelength, which can create a strong highlight, but is less than mirror reflection. The strength of echo has some relationship with the incidence angle. The shape and length of this kind of highlights can represent geometrical shape of the target, which can used in target classify and identify.

Elasticty Highlight. these highlight is formed when incident sound wave surround the surface. They are not the real geometrical highlight. The article 1 study the principle of echo impulse series of small rigid ball. The first strong echo is mirror reflect, and the other is elasticity highlight, which is small in strength.

\section{Liner model of target}

Torpedo homing system can change the width of active signal according to the distance to target, 
which can transmit short impulse at short distance. The highlight can be distinguished on some incidence angle.

\begin{tabular}{|c|c|c|c|c|}
\hline Send Signal & $\begin{array}{c}\text { Sea Sound } \\
\text { Channel }\end{array}$ & $\begin{array}{c}\text { Target } \\
\text { Response }\end{array}$ & $\begin{array}{c}\text { Sea Sound } \\
\text { Channel }\end{array}$ & $\begin{array}{c}\text { Receive } \\
\text { Signal }\end{array}$ \\
\cline { 2 - 3 }
\end{tabular}

FIG1 Transfer Process of Signal

The received signal on the torpedo homing array is the echo of transmit signal, which pass through the ocean sound channel, reflected by the target, pass through the sea sound again, and reach the torpedo at last. As shown in the figure 1. Round trip transfer lost is neglected in order to focus on the analysis of target character.

From the view of transfer model of linear system, if the incoming signal on the submarine surface is $p_{i}(r, t)$, the impulse response function of submarine is $h_{t}(r, t)$. Then the reflected signal is represented as:

$$
p_{b}(r, t)=p_{i}(r, t) * h_{t}(r, t)
$$

The major concerns on this simulation are mirror reflect and angular scattering highlight. The elasticity highlight, because of its weak strength, is looked as echo background with uniform distribution on time and space. Formula 1 can be expressed as:

$$
h_{t}(r, t)=\sum_{i=0}^{m-1} h_{0 t}\left(r_{i}, t\right)+\sum_{j=0}^{n-1} h_{1 t}\left(r_{j}, t\right)+\sum_{k=0}^{l-1} h_{r t}\left(r_{k}, t\right)
$$

In formula 2, $h_{0 t}(r, t), h_{1 t}(r, t)$ and $h_{r t}(r, t)$ respectively are impulse response functions of mirror reflect, angular scattering and elasticity highlight.

There are some characters will be found when compare the echo of underwater target with the incident sound wave: the change of wave strength, the time delay, and the phase jump, all these changes are relationship with the incident angle and incident type, which is very important to acoustic echo simulation. In addition, the Doppler shift caused by target moving should be considered.

\section{Simulation of ascoustic echo}

Normally the submarine can be seen as a spheroid, as show in the figure 2 .

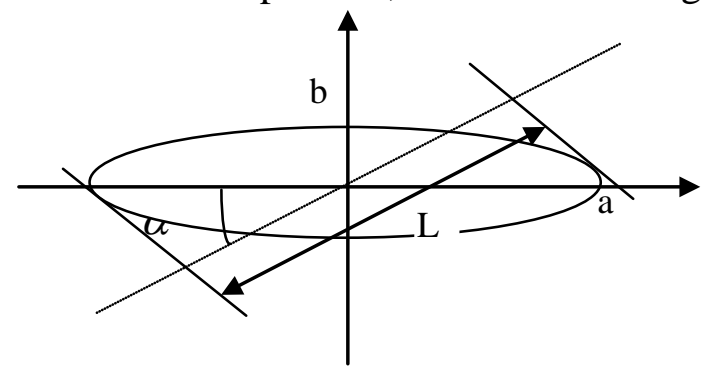

FIG2 time domain spread

When the sound signal incidents the target in the angel of $\alpha$, the time spread width is:

$$
\tau_{e}=2 \frac{L}{c}=\frac{4}{c} \sqrt{a^{2} \cos ^{2} \alpha+b^{2} \sin ^{2} \alpha}
$$

In formula 3, a, b respectively is the length of long and minor axis, and $\mathrm{c}$ is the sound speed in water. the total time spread width of reflection is:

$$
\tau_{t}=\tau_{p}+\tau_{e}=\tau_{p}+\frac{4}{c} \sqrt{a^{2} \cos ^{2} \alpha+b^{2} \sin ^{2} \alpha}
$$

In digital simulation, if the value of incident sound pressure on submarine surface is $p_{i}(t)$, then the reflected pressure is[6]: 


$$
p_{b 1}=\frac{p_{i}}{2 \sqrt{\left(1+\frac{r_{1}}{R_{1}}\right)\left(1+\frac{r_{1}}{R_{2}}\right)}} \times \sqrt{\frac{\left(1-m^{2}\right) \sin ^{2} k l}{4 m^{2}+\left(1-m^{2}\right) \sin ^{2} k l}}
$$

In formula 5, $r_{1}$ is the distance between reflected point with torpedo, $R_{1}, R_{2}$ is the radius of curvature of where the mirror reflect occurred on submarine. $m$ is the ratio of characteristic impedance between hull of submarine and sea water, $k=\omega / c$ is the wave number, $l$ is the thickness of submarine hull. When mirror reflect occurs, the phase jump $\varphi=0$.

When the angular scattering highlight occurs, the reflected pressure is:

$$
p_{b 2}=\frac{l_{1}}{4 \pi} \sqrt{\frac{\pi}{k l_{1}}} \frac{1}{\sin ^{3 / 2} \alpha \cos \alpha}
$$

In formula $6, l_{1}$ is the length of angular side. When angular scattering highlight occurs, the phase jump $\varphi=\pi / 4$,

As show in the figure 3 , in the triangle of torpedo shooting, $\mathrm{w}$ is the torpedo, $\mathrm{v} 1$ is the velocity of torpedo, $\mathrm{d}$ is the submarine, $\mathrm{v} 2$ is the velocity of target moving, $\mathrm{c}$ is the hit point, $\phi$ is lead angle, $\theta$ is the angle of impact.

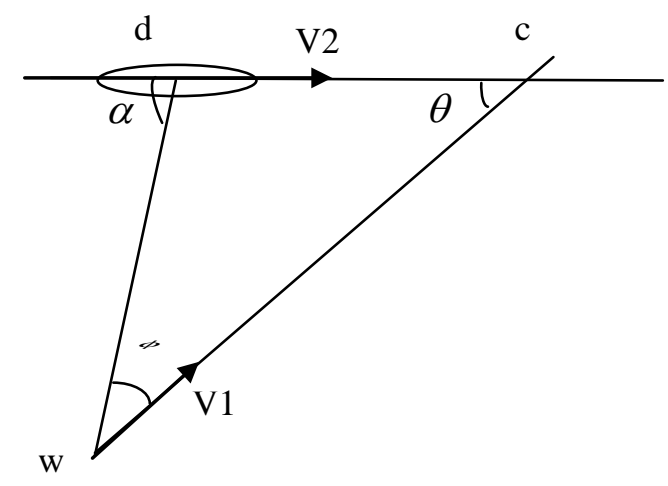

FIG3 triangle of torpedo shooting,

In the simulation, on the assumption that target's position $\left(x_{d}, y_{d}, z_{d}\right)$, the velocity $\mathrm{v} 2$ and appearance shape of target are all well known; the velocity of torpedo is $v 1$, the center position of receive array is $\left(x_{w}, y_{w}, z_{w}\right)$, the transmit beam is direct to target centre. The transmit signal is narrow-band, under these condition, all the reflected echo of highlight can be calculated.

For example, to calculate the reflected highlight signal of submarine sail, we can approximate it as elliptic cylinder. The geometric centre $\left(x_{1}, y_{1}, z_{1}\right)$ can be determined by coordinate and structure. The radius of curvature is known. We can calculate the echo strength of highlight according to formula 5 . The time delay is determined by centre position of sail and receive array:

$$
\tau_{1}=\frac{\sqrt{\left(x_{1}-x_{w}\right)^{2}+\left(y_{2}-y_{w}\right)^{2}+\left(z_{2}-z_{w}\right)^{2}}}{c}
$$

Using this method, the wave form of time domain $r(t)$ can be calculated, In the similar methods, we can get the value of angular scattering highlight. Elasticity highlight is looked as echo background with uniform distribution in $\left(\tau_{1}-\tau_{t} / 2, \tau_{1}-\tau_{t} / 2\right)$. 


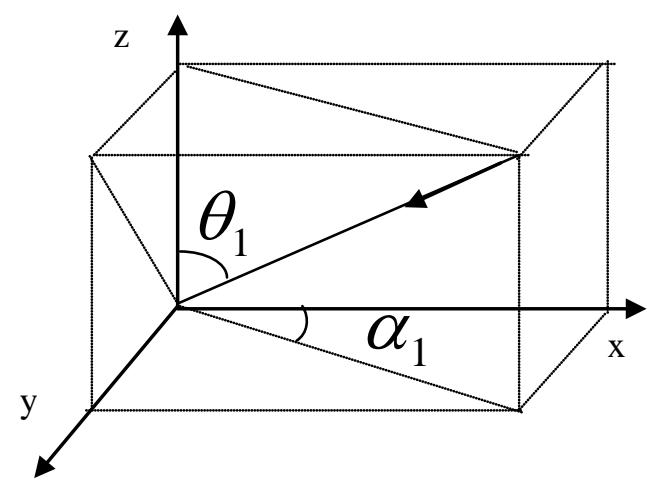

FIG4 Space angle of target

The angle information is reflected in the time-delay $\left(\Delta \tau_{i}\right)$ of signal arriving array spot. If the receive array is plane, coordinate origin is centre of array, the torpedo moving direction is the coordinate axis of $\mathrm{Z}$, then the space angle of highlight relative to coordinate system of torpedo moving is:

$$
\begin{gathered}
\theta_{1}=\arccos \frac{z_{1}-z_{w}}{r_{1}} \\
\alpha_{1}=\arctan \frac{y_{1}-y_{w}}{x_{1}-x_{w}}
\end{gathered}
$$

In formula 8, $r_{1}$ is the distance between the highlight to centre of array, then in this direction, the time delay of signal arrived at array spot $\left(x_{w i}, y_{w j}, 0\right)$ is:

$$
\tau_{w i}=\frac{x_{w i} \sin \theta_{1} \cos \alpha_{1}+y_{w i} \sin \theta_{1} \sin \alpha_{1}}{c}
$$

The output of this array spot is $r_{i}(t)=r\left(t-\tau_{w i}\right)$. We can get its value form the interpolation of the time domain signal $r(t)$ arriving array centre.

\section{Simulation result and analysis}

In simulation, the distance from torpedo to target is $500 \mathrm{~m}$, the long axis of target is $100 \mathrm{~m}$, the minor axis is $30 \mathrm{~m}$, From the result of simulation, the duration of echo is differ to the difference of incident angle. The highlight is almost can't distinguish, when the angle is great. When the angles is small, the highlights can be easily separated from each other. We can cut the short impulse to pieces to process separately, and to do some work such as character distinguishing and target classification.

\section{Conclusion}

This paper simulates the highlight of mirror reflection and angular scattering. Because of the complex construction of target, the real echo simulation should use more complex methods such as finite element analysis. At the same time, the wake and the ocean channel's effects on target echo must be added in simulation.

\section{Reference}

[1] Ji Sunxing, Zhu Ye. The identify and trace of echo highlight on near sonar [J]. Journal of acoustics. 1999; 24(4); 377-383

[2] Wang Yunping. Digit beamforming and direction of target precisely estimation [D]. master's thesis of northwest polytechnical university. 1994

[3] Jiang Yongming, Feng Haihong, Hao Xingya, Hui Junying. The Study of 2D distribution about 
underwater target [J]. Journal of acoustics; 22(1); 80-86

[4] Tang Weilin. Highlight model of target echo on sonar [J]. Journal of acoustics. 1994; 19(2); 92-100

[5] Fan Jun, Zhu Peili, Tang Weilin. The corrected geometry highlight model of echo on sonar about non-rigid surface targets [J]. Journal of acoustics. 2001; 26(6): 153-158

[6] Brill D, Gaunaurd G. Backscattering of Sound Pulse by Elastics Body [J]. Underwater Appl. acoustics.1991; 87-107 